\title{
Tactile Perceptual Dimensions: A Study with Light-Weight Wool Fabrics`
}

\author{
Alexandra Fernandes and Pedro B. Albuquerque \\ Departamento de Psicologia, Universidade do Minho \\ Campus de Gualtar, 4710-057 Braga, Portugal
}

\{alexandra.fernandes\} @gmail.com

\begin{abstract}
The aim of this study was to identify the tactile dimensions in the discrimination of light-weight wool fabrics. The participants judged the overall similarity between 21 light-weight wool fabrics using free sorting tasks. The fabrics were evaluated using active touch with limited exploratory procedure. Multidimensional scaling (MDS) was used to generate the perceptual space, revealing one dimension of tactile perception. Finally, through regression analysis we were able to interpret this dimension, using verbal attributes and physical properties of the fabrics. We discuss the relevance of the stimuli properties and the associations between verbal attributes and between physical properties, on the evaluation of the fabrics, considering its theoretical implications.
\end{abstract}

\section{Introduction}

The first systematic studies on tactile perception were reported in David Katz's book "The world of touch" 11. Katz differentiates between modifikationen - the qualities of tactile stimuli, like roughness - and spezifikationen - the identifying characteristics of the stimuli, like the silkiness of silk.

The identifying characteristics can be interpreted as a result of combinations between the stimuli objective qualities, the individual preferences, the descriptive skills of the evaluator, or even the familiarity with the stimuli. Therefore, the integration of the physical properties, and how they originate a global perception is one of the most relevant aspects in predicting the subject's tactile sensation.

Garner presented a theory concerning the integration of the stimuli properties 2. He differentiated the integral properties - related to overlaid dimensions of the objects - from the separable properties of the stimuli - well defined dimensions of the objects which are not perceptually connectible. Nonetheless, the classification of stimuli properties as integral or separable is not straightforward. There are several factors that can affect the processing of the properties, facilitating or preventing their integration [3].

\footnotetext{
* Research developed in the project COM4Wool - Design of Light-Weight Wool Fabrics; Valorization and Optimization of Total Comfort for High Added Value Clothing. Coordinated by Elisabete C. Silva, PhD. Reference: UMINHO/IDEIACOM4WOOL/BI/2/05.
} 
Researchers have tried to identify the number of tactile dimensions in the perception of familiar stimuli. Previous studies have used free sorting tasks to analyze tactile perception [4, [5, 6], 7, 8]. With this task, it is possible to represent and interpret tactile dimensions through MDS solutions using the sensory attributes or the physical properties of the stimuli. MDS studies identified two [4 to four perceptual dimensions 7 that are interpreted similarly by the authors, but with some differences according to the type of stimuli evaluated or the selected exploratory procedure.

The apparent incongruence in the number and/or nature of tactile dimensions can be related not to actual differences in the processed dimensions, but to variations in their relevance for the participant 7 . Thus, the specific properties of the stimulus and its variation in the evaluated set determine the salience of the tactile dimensions, contributing to the perceived similarity between the stimuli.

The dimensions in tactile perception are not frequently studied, and MDS techniques may improve the comprehension of the tactile perceptive organization, since they take into account several properties of the stimuli at the same time.

\section{Method}

Participants: Twenty-five volunteers (13 were female), mean age of 24.76 (SD $=3.06)$, undergraduate and graduate students from University of Minho.

Materials and Stimuli: Twenty-one fabric samples $(21 \mathrm{x} 21 \mathrm{~cm})$ of light-weight wool fabrics with differences in torsion ( $\mathrm{Z}$ or S), structure (taffeta, twill weave, and twill weave derivate) and finishing (dyed yarn or dyed piece). For the evaluation of the eight verbal attributes - delicate, thick, light, smooth, soft, flexible, warm, and resistant - the participants were presented with a $12.7 \mathrm{~cm}$ (5 inches) graphic scale, marked in the poles: "nothing" $(0 \mathrm{~cm})$ and "very" $(12.7 \mathrm{~cm})$. The participants were instructed to mark the point in the scale that better translated their sensation when touching each fabric. These verbal attributes were selected through previous vocabulary procedures $[9$. To block the vision of the fabrics during the task, we used a white placard with two openings for the hands. The participants wore a rubber glove, with the thumb and index finger completely removed. The participants washed their hands with glycerin soap and dried it with paper towels before the task, and whenever they needed during the procedure.

Procedure: Each participant completed a free sorting task with the 21 fabric samples, repeated in two sessions, in consecutive weeks ( $M=7.0$ days, $S D=2.7$ days), without training session. The participants wore the rubber glove on the dominant hand while evaluating the fabrics, and were allowed to rest during the evaluation. They were instructed to create groups of fabric samples based on similarity, with no restrictions to the number of groups formed or to the number of samples allowed in each group. The fabric samples were presented randomly and individually, with no limitation of time of contact. The first sample was 
presented as Sample 1 and it represented the A Group. The second sample, presented as Sample 2, could be added to A Group (if evaluated as similar to Sample 1), or used to form the B Group (if evaluated as different from Sample 1). After the evaluation of all the samples, the generated groups were presented to the participant, following the same order by which the fabric samples were gathered in each group. The participants were allowed to touch any sample at any time during the evaluation, but always one sample at a time. Before the final presentation of the groups, the participants were told they could join or divide the previously formed groups in order to reach a better representation of their tactile sensation. At the end of the second session, after the free sorting task, the participants evaluated the verbal attributes.

\section{Results}

The participants formed between 2 and 6 groups of fabric samples $(M=3.62$; $S D=1.05 ; M o d e=4)$. Sixty percent of the participants maintained the number of groups in both sessions, and the consistency in the sorting task (percentage of times each pair of fabrics was grouped together on both sessions) ranged between $55.1 \%$ and $78.2 \%(M=67.6 \%, S D=5.97 \%)$.

The MDS revealed that the adjustment measures $1-R^{2}$ and Stress had acceptable levels in the one-dimensional solution [10]. The $1-R^{2}$ values explain $96 \%$ of the variability and the Stress value is 0.12 . These measures do not improve in the two-dimensional representation, indicating that the one-dimensional solution is reliable. Figure 1 represents the one-dimensional solution. Two close points reveal tactile similarities between the represented fabric samples. Some samples are overlaid - A1, A2 and A10; A9, A11 and A12; A15 and A19; A4, A16 and A20 - indicating that they were perceived as identical by the evaluators.

The verbal attributes were measured in centimeters from the "zero point" on the scale, associated with the "nothing" pole. The participants were able to

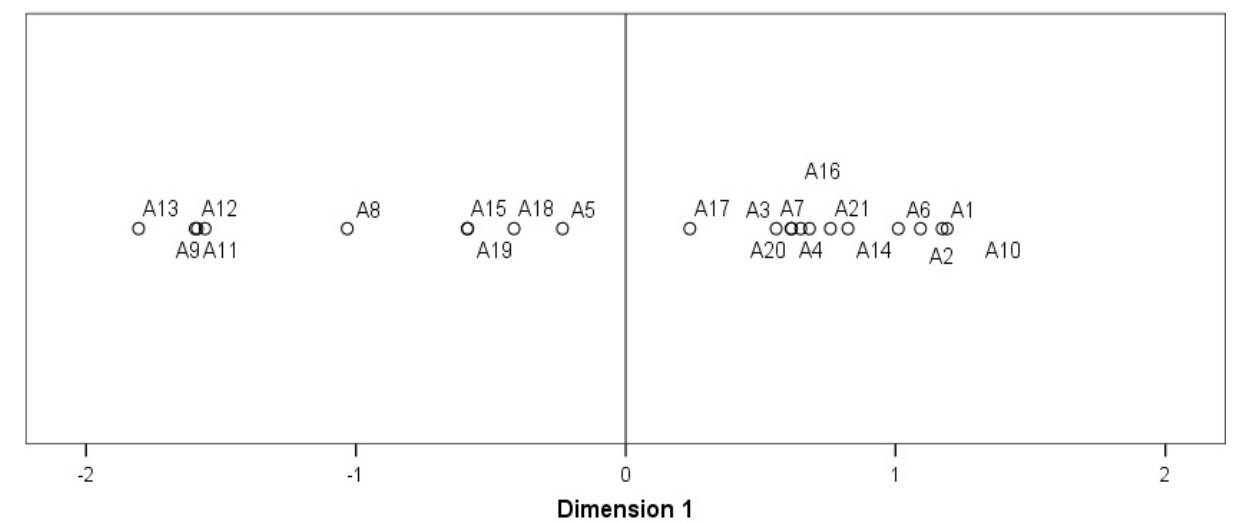

Fig. 1. Representation of the one-dimensional solution from MDS 
Table 1. Verbal atributtes' correlation matrix $\left({ }^{*} p<0.01\right)$

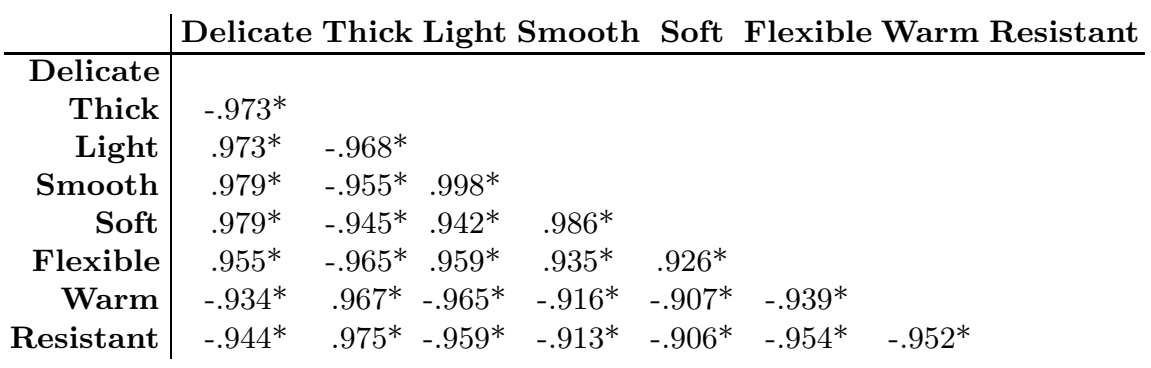

differentiate all the attributes, as shown by the ANOVA analysis of the individual evaluations: delicate $(F(20,504)=13.10, p<0.01)$; thick $(F(20,504)=18.43, p<$ $0.01)$; light $(F(20,503)=14.02, p<0.01)$; smooth $(F(20,504)=16.29, p<0.01)$; soft $(F(20,504)=15.99, p<0.01)$; flexible $(F(20,504)=11.53, p<0.01)$; warm $(F(20,504)=8.90, p<0.01)$; and resistant $(F(20,504)=5.64, p<0.01)$. The difference between the fabric with the highest and the fabric with the lowest scale value for each attribute varies from 4.5 (resistant) to 7.4 (smooth), with a mean variation of $6.2(S D=0.95)$.

The correlation matrix for the verbal attribute scales is shown in table 1 . Results show as strong association between the evaluations of the attributes, originating two groups (based on the type of correlations - negative and positive): one composed by the attributes thick, warm, and resistant and other by the remaining attributes.

To interpret the MDS solution, the mean evaluations of the attributes for each fabric were regressed into the coordinates of each stimulus on the MDS solution (table 2). The $\beta$ values are very high for all the attributes, revealing a strong association of the attributes with the one-dimensional space. The attribute soft presents the higher $\beta$ value (0.978), followed by the attributes delicate and smooth, all surface attributes. The attributes thick, warm and resistant are negatively associated with the perceptual dimension, while the others present positive relations. Although the attributes had well distinguishable meanings 9], all revealed to be related to the one-dimensional perceptual solution.

The physical properties present a tight relationship in this set of stimuli, with significant correlations (as shown in table 3 ), contributing to the one-dimensional pattern in the MDS solution.

Table 4 shows the $\beta$ coefficients for the physical properties, resultant from its regression in the MDS coordinates. The ligament coefficient (fabric tightness), thickness, weft density, weight, and warp and weft yarn title (thickness of the

Table 2. Standardized $\beta$ values for the verbal attributes $\left({ }^{*} p<0.01\right)$

Delicate Thick Light Smooth Soft Flexible Warm Resistant

\begin{tabular}{l|llllllll}
\hline$\beta$ Values & $.976^{*}$ & $-.948^{*}$ & $.955^{*}$ & $.973^{*}$ & $.978^{*}$ & $.934^{*}$ & $-.898^{*}$ & $-.908^{*}$
\end{tabular}


Table 3. Physical properties' correlation matrix $\left({ }^{*} p<0.01 ;{ }^{* *} p<0.05\right)$

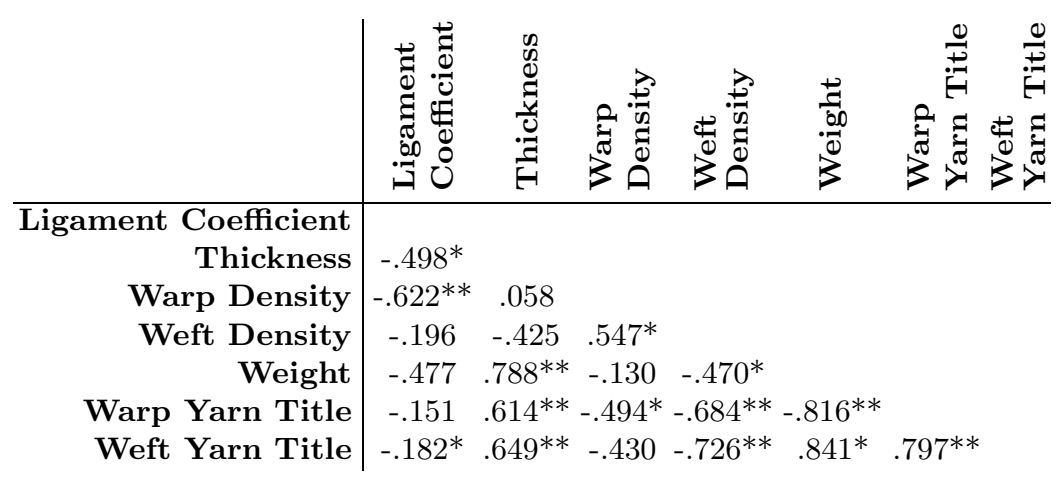

Table 4. Standardized $\beta$ values for the physical properties $\left({ }^{*} p<0.01\right.$; $\left.^{* *} p<0.05\right)$

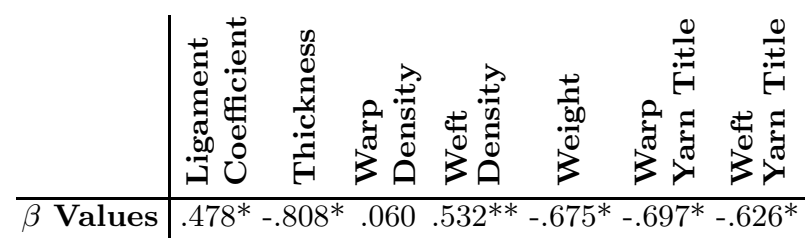

yarn) are significantly associated with the perceptual dimension - the properties ligament coefficient and weft density are positively related to the perceptual dimension, while the other present negative associations. The overall $\beta$ values of the physical properties are lower than the verbal attributes values. This may be interpreted as an indication that each physical property by itself cannot explain the MDS solution. The verbal attributes could, therefore, describe a more complex perception of the stimuli.

\section{Conclusions}

With this study we were able to define an one-dimensional solution in the tactile perception of light-weight wool fabrics. This solution appears to be a good translation of the participants' sensations and represents a robust dimension. Both physical properties and verbal attributes are projected in the dimensional space, confirming the one-dimensional solution, since interpretability is a relevant criterion for the dimensional decision in MDS [10].

In this set of fabrics, it is possible to preview the consumers' evaluations, based on two attribute groups (positive and negative), which are conceptually linked to the same perceptual dimension.

Through a procedure which did not involve experts, we were able to define a global and stable sensory profile in a short period of time. The defined dimension of light-weight wool fabrics is positively associated with the attributes delicate, 
light, smooth, soft, and flexible and with the physical properties ligament coeffcient and weft density; and negatively related to the attributes thick, warm, and resistant, and the properties thickness, weight, and weft/warp yarn title.

Although the participants were able to differentiate the fabrics, they compose a tactually similar set, discriminated essentially by global sensations. The MDS dimension may be interpreted as an identifying characteristic of the lightweight wool fabrics, implying all the verbal attributes. However, in a qualitative analysis, this dimension is verbalized exclusively as surface properties by the participants, particularly the attribute soft.

\section{References}

1. Katz, D.: The World of Touch (L. E. Krueger, Trans.). Lawrence Erlbaum, Hillsdale (1989) (Original work published in 1925)

2. Garner, W.R.: The Processing of Information and Structure. Wiley, New York (1974)

3. Klatzky, R.L., Lederman, S.J., Reed, C.: Haptic Integration of Object Properties: Texture, Hardness, and Planar Contour. Journal of Experimental Psychology: Human Perception and Performance 15, 45-57 (1989)

4. Hollins, M., Faldowski, R., Rao, S., Young, F.: Perceptual Dimensions of Tactile Surface Texture: A Multidimensional Scaling Analysis. Perception and Psychophysics 56, 697-705 (1993)

5. Hollins, M., Bensmaïa, S., Karlof, K., Young, F.: Individual Differences in Perceptual Space for Tactile Textures: Evidence from Multidimensional Scaling. Perception and Psychophysics 62, 1534-1544 (2000)

6. Hollins, M., Lorenz, F., Seeger, A., Taylor, R.: Factors Contributing to the Integration of Textural Qualities: Evidences from Virtual Surfaces. Somatossensory and Motor Research 22, 193-206 (2005)

7. Picard, D., Dacremont, C., Valentin, D., Giboreau, A.: Perceptual Dimensions of Tactile Textures. Acta Psychologica. 114, 165-184 (2003)

8. Tiest, W.M., Kappers, A.M.: Analysis of Haptic Perception of Materials by Multidimensional Scaling and Physical Measurements of Roughness and Compressibility. Acta psychologica. 121, 1-20 (2006)

9. Fernandes, A.: Identification of the perceptual dimensions in tactile differentiation of light-weight wool fabrics. Universidade do Minho: Departamento de Psicologia (2007) [unpublished thesis]

10. Kruskal, J.B., Wish, M.: Multidimensional Scaling. Sage, Beverley Hills (1978) 